\title{
Dynamic Inequalities in Quotients with General Kernels and Measures
}

\author{
H. A. Abd El-Hamid, ${ }^{1}$ H. M. Rezk, ${ }^{2}$ A. M. Ahmed $\left(\mathbb{D},{ }^{2,3}\right.$ Ghada AlNemer ${ }^{\mathbb{D}},{ }^{4}$ M. Zakarya ${ }^{(D)},{ }^{5,6}$ \\ and H. A. El Saify ${ }^{1}$
}

${ }^{1}$ Department of Mathematics and Computer Science, Faculty of Science, Beni-Suef University, Beni Suef, Egypt

${ }^{2}$ Department of Mathematics, Faculty of Science, Al-Azhar University, Nasr City 11884, Egypt

${ }^{3}$ Mathematics Department, College of Science, Jouf University, Sakaka (2014), Saudi Arabia

${ }^{4}$ Department of Mathematical Science, College of Science, Princess Nourah bint Abdulrahman University, P.O. Box 105862,

Riyadh 11656, Saudi Arabia

${ }^{5}$ King Khalid University, College of Science, Department of Mathematics, P.O. Box 9004, 61413 Abha, Saudi Arabia

${ }^{6}$ Department of Mathematics, Faculty of Science, Al-Azhar University, 71524 Assiut, Egypt

Correspondence should be addressed to Ghada AlNemer; gnnemer@pnu.edu.sa

Received 5 June 2020; Accepted 14 July 2020; Published 3 August 2020

Guest Editor: Lishan Liu

Copyright (C) 2020 H. A. Abd El-Hamid et al. This is an open access article distributed under the Creative Commons Attribution License, which permits unrestricted use, distribution, and reproduction in any medium, provided the original work is properly cited.

By utilizing the precepts related to the punctuation of time scales $\mathbb{T}$, we present some nouveau forms in quotients for Hardy's and related inequalities on time scales. In particular, some recent results for the Pólya-Knopp, Hardy-Hilbert, and Hardy-LittlewoodPólya-type inequalities are presented.

\section{Introduction}

In [1], Hardy stated and proved the following integral inequality:

$$
\int_{0}^{\infty}\left(\theta^{-1} \int_{0}^{\theta} f(\eta) d \eta\right)^{\xi} d \theta \leq\left(\frac{\xi}{\xi-1}\right)^{\xi} \int_{0}^{\infty} f^{\xi}(\theta) d \theta, \quad \text { for } \xi>1,
$$

where $f$ is a positive function and the constant $(\xi /(\xi-1))^{\xi}$ is the best ever. We rewrite (1) with the function $f^{\xi^{-1}}$ rather than $f$, and by assuming limit $\xi \rightarrow \infty$, we acquired the limiting instance of the inequality of Hardy known as the inequality of Pólya-Knopp (see [2]), that is,

$$
\int_{0}^{\infty} \exp \left(\theta^{-1} \int_{0}^{\theta} \ln f(\eta) d \eta\right) d \theta \leq e \int_{0}^{\infty} f(\theta) d \theta .
$$

Lately, Kaijser et al. in [3] pointed out that both (1) and (2) are just special states of the much more general inequality of Hardy-Knopp for positive function $f$ :

$$
\int_{0}^{\infty} \Phi\left(\theta^{-1} \int_{0}^{\theta} f(\eta) d \eta\right) \frac{d \theta}{\theta} \leq \int_{0}^{\infty} \Phi(f(\theta)) \frac{d \theta}{\theta},
$$

where $\Phi \in C((0, \infty), \mathbb{R})$ is a convex function. A popularization of inequality (3) with two weight functions is proved in [4]. Particularly, it was proved that if $0<b \leq \infty, \lambda:(0, b)$ $\rightarrow \mathbb{R}$ is a nonnegative function and $\Phi$ is a convex on $(a, c)$, then the inequality

$$
\int_{0}^{b} \lambda(\theta) \Phi\left(\frac{1}{\theta} \int_{0}^{\theta} f(\eta) d \eta\right) \frac{d \theta}{\theta} \leq \int_{0}^{b} v(\theta) \Phi(f(\theta)) \frac{d \theta}{\theta},
$$

is available for all integrable functions $f:(0, b) \rightarrow \mathbb{R}$, such that $f(\theta) \in(a, \mathrm{c})$ and $v$ is defined by 


$$
v(\eta):=\eta \int_{\eta}^{b} \frac{u(\theta)}{\theta^{2}} d \theta, \quad \text { for } \eta \in(0, b)
$$

Using the inequality of Jensen for convex functions and the theorem of Fubini, Kaijser et al. [5] established an inviting popularization of (1). Particularly, they proved that if $\lambda:(0, b) \rightarrow \mathbb{R}$ and $k:(0, b) \times(0, b) \rightarrow \mathbb{R}$ are nonnegative functions such that $0<K(\theta)=\int_{0}^{\theta} k(\theta, \eta) d \eta<\infty$, for $\theta \in(0, b)$ and

$$
v(\eta):=\eta \int_{\eta}^{b} \lambda(\theta) \frac{k(\theta, \eta)}{K(\theta)} \frac{d \theta}{\theta}<\infty, \quad \text { for } \eta \in(0, b),
$$

then

$$
\int_{0}^{\infty} \lambda(\theta) \Phi\left(A_{k} f(\theta)\right) \frac{d \theta}{\theta} \leq \int_{0}^{\infty} v(\theta) \Phi(f(\theta)) \frac{d \theta}{\theta}
$$

where $\Phi \in \mathrm{C}(I, \mathbb{R})$ is a convex function and

$$
A_{k} f(\theta):=\frac{1}{K(\theta)} \int_{0}^{\theta} k(\theta, \eta) f(\eta) d \eta, \quad \theta \in(0, b)
$$

For further popularization of (7), Krulić et al. [6] proved that if $\left(\Omega_{1}, \Sigma_{1}, \mu_{1}\right)$ and $\left(\Omega_{2}, \Sigma_{2}, \mu_{2}\right)$ are two measure spaces with positive $\sigma$-finite measures $\lambda: \Omega_{1} \rightarrow \mathbb{R}$ and $k: \Omega_{1} \times \Omega_{2} \rightarrow \mathbb{R}$ which are nonnegative measurable functions such that $0<K(\theta)=\int_{\Omega_{2}} k(\theta, \eta) d \mu_{2}(\eta)<\infty, \theta \in \Omega_{1}$, $0<\xi \leq \xi^{*}<\infty$, and $v$ is defined by

$$
\nu(\eta):=\left(\int_{\Omega_{1}} \lambda(\theta)\left(\frac{k(\theta, \eta)}{K(\theta)}\right)^{\xi^{*} / \xi} d \mu_{1}(\theta)\right)^{\xi / \xi^{*}}<\infty, \quad \eta \in \Omega_{2}
$$

then the inequality

$$
\left(\int_{\Omega_{1}} \lambda(\theta) \Phi^{\xi^{*} / \xi}\left(A_{k} f(\theta)\right) d \mu_{1}(\theta)\right)^{1 / \xi^{*}} \leq\left(\int_{\Omega_{2}} v(\eta) \Phi(f(\eta)) d \mu_{2}(\eta)\right)^{1 / \xi}
$$

is available for all measurable functions $f: \Omega_{2} \rightarrow \mathbb{R}$ such that $f\left(\Omega_{2}\right) \subseteq I$, where $\Phi \in \mathrm{C}(I, \mathbb{R})$ is a convex function and $A_{k}$ is defined by

$$
A_{k} f(\theta):=\frac{1}{K(\theta)} \int_{\Omega_{2}} k(\theta, \eta) f(\eta) d \mu_{2}(\eta), \theta \in \Omega_{1}
$$

In [7], Iqbal et al. checked some new weighted Hardytype inequalities on $\left(\Omega_{1}, \Sigma_{1}, \mu_{1}\right)$ and $\left(\Omega_{2}, \Sigma_{2}, \mu_{2}\right)$ measure spaces with positive $\sigma$-finite measures by replacing $k(\theta, \eta)$ by $k(\theta, \eta) f_{2}(\theta)$ and $f$ by $f_{1} / f_{2}$, where $f_{i}: \Omega_{2} \rightarrow \mathbb{R}(i=1,2)$ are measurable functions in (10) as follows:

$$
\begin{gathered}
\left(\int_{\Omega_{1}} \lambda(\theta)\left[\Phi\left(\frac{g_{1}(\theta)}{g_{2}(\theta)}\right)\right]^{\xi^{*} / \xi} d \mu_{1}(\theta)\right)^{1 / \xi^{*}} \\
\leq\left(\int_{\Omega_{2}} v(\eta) \Phi\left(\frac{f_{1}(\eta)}{f_{2}(\eta)}\right) d \mu_{2}(\eta)\right)^{1 / \xi},
\end{gathered}
$$

where $0<\xi \leq \xi^{*}<\infty, \Phi \in \mathrm{C}(I, \mathbb{R})$ is a convex function, $k: \Omega_{1} \times \Omega_{2} \rightarrow \mathbb{R}$ is a nonnegative measurable function, and $v, g_{i}(\theta)(i=1,2)$ are defined by

$$
\begin{aligned}
& v(\eta):=f_{2}(\eta)\left(\int_{\Omega_{1}} \lambda(\theta)\left(\frac{k(\theta, \eta)}{g_{2}(\theta)}\right)^{\xi^{*} / \xi} d \mu_{1}(\theta)\right)^{\xi / \xi^{*}}<\infty \\
& g_{2}(\theta) \neq 0, \eta \in \Omega_{2}, \\
& g_{i}(\theta):=\int_{\Omega_{2}} k(\theta, \eta) f_{i}(\eta) d \mu_{2}(\eta) \quad(i=1,2) .
\end{aligned}
$$

In [8], the author founded the time scale version of (1). Particularly, he proved that if $\xi>1, f \in \mathrm{C}_{r d}\left(\left[a_{1}, \infty\right]_{\mathbb{T}}, \mathbb{R}\right)$ is a nonnegative function and $\int_{a_{1}}^{\infty} f^{\xi}(t) \Delta t$ exists, then

$$
\int_{a_{1}}^{\infty}\left(\frac{1}{\sigma(t)-a_{1}} \int_{a_{1}}^{\sigma(t)} f(\theta) \Delta \theta\right)^{\xi} \Delta \eta \leq\left(\frac{\xi}{\xi-1}\right)^{\xi} \int_{a_{1}}^{\infty} f^{\xi}(\eta) \Delta \eta .
$$

In [9], the authors proved the time scale version of (4), which is given by

$$
\int_{a_{1}}^{b_{1}} \lambda(\theta) \Phi\left(\frac{1}{\sigma(\theta)-a_{1}} \int_{a_{1}}^{\sigma(\theta)} f(\eta) \Delta \eta\right) \frac{\Delta \theta}{\theta-a_{1}} \leq \int_{a_{1}}^{b_{1}} v(\theta) \Phi(f(\theta)) \frac{\Delta \theta}{\theta-a_{1}}
$$

where $\Phi \in \mathrm{C}\left(\left(c_{1}, d_{1}\right), \mathbb{R}\right)$ is a convex function, $\lambda \in \mathrm{C}_{r d}$ $\left(\left[a_{1}, b_{1}\right]_{\mathbb{T}}, \mathbb{R}\right)$ is a nonnegative function, and $v$ is defined by

$v(t):=\left(\eta-a_{1}\right) \int_{t}^{b_{1}} \frac{\lambda(\eta)}{\left(\theta-a_{1}\right)\left(\sigma(\theta)-a_{1}\right)} \Delta \theta, \quad$ for $\eta \in\left(a_{1}, b_{1}\right)_{\mathbb{T}}$.

In [10], the authors outstretched a number of Hardy-type inequalities with certain kernels on time scale. Namely, they proved that if $\left(\Omega_{1}, \Sigma_{1}, \mu_{1}\right)$ and $\left(\Omega_{2}, \Sigma_{2}, \mu_{2}\right)$ are two time scale measure spaces, $\zeta: \Omega_{1} \rightarrow \mathbb{R}_{+}$and $k: \Omega_{1} \times \Omega_{2} \rightarrow \mathbb{R}_{+}$, which are nonnegative measurable functions such that

$$
K(\theta):=\int_{\Omega_{2}} k(\theta, \eta) \Delta \eta<\infty, \quad \theta \in \Omega_{1},
$$


and $v$ is defined by

$$
v(\eta):=\int_{\Omega_{1}} \frac{k(\theta, \eta) \zeta(\theta)}{K(\theta)} \Delta \theta<\infty, \quad \eta \in \Omega_{2},
$$

then the inequality

$$
\int_{\Omega_{1}} \zeta(\theta) \Phi\left(\frac{1}{K(\theta)} \int_{\Omega_{2}} k(\theta, \eta) f(\eta) \Delta \eta\right) \Delta \theta \leq \int_{\Omega_{2}} v(\eta) \Phi(f(\eta)) \Delta \eta
$$

is available for all $\Delta \mu_{2}$-integrable $f: \Omega_{2} \rightarrow \mathbb{R}$ such that $f\left(\Omega_{2}\right) \subset I$ and $\Phi \in \mathrm{C}(I, \mathbb{R})$ is a convex function. For development of dynamic inequalities on time scale calculus, we refer the reader to articles [11-18].

The article is regimented as follows. In Section 2, we recall the precepts related to the punctuation of time scales. In Section 3, we prove our results and give some remarks. Particularly, we prove a general dynamic weighted Hardytype inequality with a nonnegative kernel. In Section 4, we critique a few particular states of the obtained inequalities, related to power and exponential functions and to the most simplest shapes of kernels.

\section{Preliminaries}

In this section, we will premise some fundamental precepts and effects on time scales which will be beneficial for deducing our major results. The following definitions and theorems are referred from $[19,20]$.

A nonempty arbitrary locked subplot of the real numbers $\mathbb{R}$ is called a time scale which is denoted by $\mathbb{T}$. For $\tau \in \mathbb{T}$, if $\inf \Phi=\sup \mathbb{T}$ and sup $\Phi=\inf \mathbb{T}$, then the forward jump operator $\sigma: \mathbb{T} \rightarrow \mathbb{T}$ and the backward jump operator $\rho: \mathbb{T}$ $\rightarrow \mathbb{T}$ are defined as

$$
\begin{aligned}
& \sigma(\tau):=\inf \{\theta \in \mathbb{T}: \theta>\tau\}, \\
& \rho(\tau):=\sup \{\theta \in \mathbb{T}: \theta<\tau\},
\end{aligned}
$$

respectively. The $\Delta$-derivative of $\chi: \mathbb{T} \rightarrow \mathbb{R}$ at $\tau \in \mathbb{T}^{k}=\mathbb{T} /(\rho$ $(\sup \mathbb{T})$, sup $\mathbb{T}]$ is the number that enjoys the property that for all $\varepsilon>0$, there exists a neighborhood $U \subset \mathbb{T}$ of $\tau \in \mathbb{T}^{k}$ such that

$\left|[\chi(\sigma(\tau))-\chi(\theta)]-\chi^{\Delta}(\tau)[\sigma(\tau)-\theta]\right| \leq \varepsilon|\sigma(\tau)-\theta|, \quad$ for all $\theta \in U$

Furthermore, $\chi$ is called a delta differentiable on $\mathbb{T}^{k}$ if it is delta differentiable at every $\tau \in \mathbb{T}^{k}$. Similarly, for $\tau \in \mathbb{T}_{k}$, we define the $\nabla$-derivative of $\chi: \mathbb{T} \rightarrow \mathbb{R}$ at $\tau \in \mathbb{T}_{k}=\mathbb{T} /(\inf \mathbb{T}, \sigma$ (inf $\mathbb{T})]$ as the number that enjoys the property that for all $\varepsilon>0$, there exists a neighborhood $V \subset \mathbb{T}$ of $\tau \in \mathbb{T}_{k}$ such that

$\left|\chi(\rho(\tau))-\chi(\theta)-\chi^{\nabla}(\tau)(\rho(\tau)-\theta)\right| \leq \varepsilon|\rho(\tau)-\theta|, \quad$ for all $\theta \in V$.
Moreover, $\chi$ is called a nabla differentiable on $\mathbb{T}_{k}$ if it is nabla differentiable at every $\tau \in \mathbb{T}_{k}$. For $\theta, t \in \mathbb{T}^{k}$, the delta integral of $\chi^{\Delta}$ is defined as

$$
\int_{\theta}^{t} \chi^{\Delta}(\tau) \Delta \tau:=\chi(t)-\chi(\theta)
$$

Similarly, for $\theta, t \in \mathbb{T}_{k}$, the nabla integral of $\chi^{\nabla}$ is defined as

$$
\int_{\theta}^{t} \chi^{\nabla}(\tau) \nabla \tau:=\chi(t)-\chi(\theta)
$$

Now, let $\chi(\tau)$ be differentiable on $\mathbb{T}$ in the $\Delta$ and $\nabla$ senses. For $\tau \in \mathbb{T}_{k}^{k}$, where $\mathbb{T}_{k}^{k}=\mathbb{T}^{k} \cap \mathbb{T}_{k}$, the diamond- $\alpha$ dynamic derivative $\chi^{\diamond_{\alpha}}(\tau)$ is defined by

$$
\chi^{\diamond_{\alpha}}(\tau):=\alpha \chi^{\Delta}(\tau)+(1-\alpha) \chi^{\nabla}(\tau), \quad 0 \leq \alpha \leq 1
$$

The diamond- $\alpha$ derivative debases to the standard $\Delta$ -derivative for $\alpha=1$ or the standard $\nabla$-derivative for $\alpha=0$.

Next, we recall the inequality of Minkowski and the inequality of Jensen on time scales which are utilized in the proof of the major results.

Theorem 1. Suppose $\left(\Omega, \mathscr{M}, \mu_{\Delta}\right)$ and $\left(\Lambda, \mathscr{L}, \lambda_{\Delta}\right)$ are two finite-dimensional time scale measure spaces, and let $u, v$, and $g$ be positive functions on $\Omega, \Lambda$, and $\Omega \times \Lambda$, respectively. If $\xi \geq 1$, then the inequality

$$
\begin{aligned}
& {\left[\int_{\Omega}\left(\int_{\Lambda} g(\theta, \eta) v(\eta) d v_{\Delta}(\eta)\right)^{\xi} u(\theta) d \mu_{\Delta}(\theta)\right]^{1 / \xi}} \\
& \quad \leq \int_{\Lambda}\left(\int_{\Omega} g^{\xi}(\theta, \eta) u(\theta) d \mu_{\Delta}(\theta)\right) v(\eta) d v_{\Delta}(\eta),
\end{aligned}
$$

is available for all integrals in (26). If $0<\xi<1$ and

$$
\begin{array}{r}
\int_{\Omega}\left(\int_{\Lambda} g v d v_{\Delta}\right)^{\xi} u d \mu_{\Delta}>0 \\
\int_{\Lambda} g v d v_{\Delta}>0
\end{array}
$$

are available, then (26) is reversed. For $\xi<0$, in addition with (27), if

$$
\int_{\Omega} g^{\xi} u d \mu_{\Delta}>0
$$

is available, then again (26) is reversed.

Theorem 2. Let $e, l \in \mathbb{T}$ and $u, v \in \mathbb{R}$. Suppose that $g_{1} \in$ $C_{r d}\left([e, l]_{\mathbb{T}},(u, v)_{\mathbb{T}}\right)$ and $h_{1} \in C_{r d}\left([e, l]_{\mathbb{T}}, \mathbb{R}\right)$ are nonnegative with $\int_{e}^{l} h_{1}(s) \Delta s>0$. If $\Phi \in C((u, v), \mathbb{R})$ is convex, then 


$$
\Phi\left(\frac{\int_{e}^{l} h_{1}(s) g_{1}(s) \Delta s}{\int_{e}^{l} h_{1}(s) \Delta s}\right) \leq \frac{\int_{e}^{l} \Phi\left(h_{1}(s)\right) g_{1}(s) \Delta s}{\int_{e}^{l} h_{1}(s) \Delta s} .
$$

\section{Inequalities with General Kernels}

In this section, we state and prove our major results. Before presenting the results, we labeled the following hypotheses.

(H1) $\left(\Omega_{1}, \Sigma_{1}, \mu_{1}\right)$ and $\left(\Omega_{2}, \Sigma_{2}, \mu_{2}\right)$ are two time scale measure spaces

(H2) $k: \Omega_{1} \times \Omega_{2} \rightarrow \mathbb{R}$ is a positive measurable kernel and

$$
K(\theta):=\int_{\Omega_{2}} k(\theta, \eta) \Delta \mu_{2}(\eta)<\infty, \quad \theta \in \Omega_{1} .
$$

(H3) $u: \Omega_{1} \rightarrow \mathbb{R}$ is a $\Delta \mu_{2}$-integrable and

$v(\eta):=\left(\int_{\Omega_{1}} u(\theta)\left(\frac{k(\theta, \eta)}{K(\theta)}\right)^{\xi^{*} / \xi} \Delta \mu_{1}(\theta)\right)^{\xi / \xi^{*}}<\infty$

where $0<\xi \leq \xi^{*}<\infty$.

In what follows, we will prove the foundation theorem that will be the decisive step in establishing our major result.

Theorem 3. Assume (H1)-(H3).

If $\Phi \in C(I, \mathbb{R})$ is a positive convex, then the following inequality

$$
\begin{aligned}
& \left(\int_{\Omega_{1}} u(\theta)\left(\Phi\left(A_{k}(f(\theta))\right)^{\xi^{*} / \xi} \Delta \mu_{1}(\theta)\right)^{1 / \xi^{*}}\right. \\
& \leq\left(\int_{\Omega_{2}} v(\eta) \Phi(f(\eta)) \Delta \mu_{2}(\eta)\right)^{1 / \xi}
\end{aligned}
$$

is available for all nonnegative $\Delta \mu_{2}$-integrable function $f: \Omega_{2} \rightarrow \mathbb{R}$ such that $\operatorname{Imf} \subseteq I$, where $A_{k} f: \Omega_{1} \rightarrow \mathbb{R}$ is defined by

$$
A_{k} f(\theta):=\frac{1}{K(\theta)} \int_{\Omega_{2}} k(\theta, \eta) f(\eta) \Delta \mu_{2}(\eta), \quad \theta \in \Omega_{1} .
$$

Proof. We begin with an evident identity

$$
\begin{array}{rl}
\int_{\Omega_{1}} & u(\theta)\left(\Phi\left(A_{k}(f(\theta))\right)^{\xi^{*} / \xi} \Delta \mu_{1}(\theta)\right. \\
\quad & \int_{\Omega_{1}} u(\theta)\left(\Phi\left(\frac{1}{K(\theta)} \int_{\Omega_{2}} k(\theta, \eta) f(\eta) \Delta \mu_{2}(\eta)\right)\right)^{\xi^{*} / \xi} \Delta \mu_{1}(\theta) .
\end{array}
$$

Utilizing the inequality of Jensen (29) and the theorem of Minkowski (2) on (34), we find that

$$
\begin{aligned}
& \int_{\Omega_{1}} u(\theta)\left(\Phi\left(A_{k}(f(\theta))\right)^{\xi^{*} / \xi} \Delta \mu_{1}(\theta)\right. \\
& \quad \leq \int_{\Omega_{1}} u(\theta)\left(\frac{1}{K(\theta)} \int_{\Omega_{2}} k(\theta, \eta) \Phi(f(\eta)) \Delta \mu_{2}(\eta)\right)^{\xi^{*} / \xi} \Delta \mu_{1}(\theta) \\
& \quad=\left(\left(\int_{\Omega_{1}} u(\theta)\left(\frac{1}{K(\theta)} \int_{\Omega_{2}} k(\theta, \eta) \Phi(f(\eta)) \Delta \mu_{2}(\eta)\right)^{\xi^{*} / \xi} \Delta \mu_{1}(\theta)\right)^{\xi / \xi^{*}}\right)^{\xi^{*} / \xi} \\
& \quad \leq\left(\int_{\Omega_{2}} \Phi(f(\eta))\left(\int_{\Omega_{1}} u(\theta)\left(\frac{k(\theta, \eta)}{K(\theta)}\right)^{\xi^{*} / \xi} \Delta \mu_{1}(\theta)\right)^{\xi / \xi^{*}} \Delta \mu_{2}(\eta)\right)^{\xi^{*} / \xi} .
\end{aligned}
$$

Taking into computation definition (31) of $v$, it follows that

$$
\int_{\Omega_{1}} u(\theta)\left(\Phi\left(A_{k}(f(\theta))\right)^{\xi^{*} / \xi} \Delta \mu_{1}(\theta) \leq\left(\int_{\Omega_{2}} v(\eta) \Phi(f(\eta)) \Delta \mu_{2}(\eta)\right)^{\xi^{*} / \xi} .\right.
$$
(33).

Finally, elevating (36) to the $\xi^{*}$ th power, we acquired

Remark 4. For the Lebesgue scale measures $\Delta \mu_{1}(\theta)=\Delta \theta, \Delta$ $\mu_{2}(\eta)=\Delta \eta$, and $\xi=\xi^{*}=1$, inequality (32) reduces to inequality (19) premised in Section 1 . Choosing $\mathbb{T}=\mathbb{R}$ in Theorem 3, then inequality (32) reduces to inequality (10).

In what follows, we labeled few particular convex functions starting with power functions.

Corollary 5. Suppose the supposition of Theorem 3 be gratified only with $\Phi(x)=x^{\xi}$ for $\xi>1$. Then, inequality (32) yields the following result:

$$
\left(\int_{\Omega_{1}} u(\theta)\left[A_{k}(f(\theta)]^{\xi^{*}} \Delta \mu_{1}(\theta)\right)^{1 / \xi^{*}} \leq\left(\int_{\Omega_{2}} v(\eta)[f(\eta)]^{\xi} \Delta \mu_{2}(\eta)\right)^{1 / \xi} .\right.
$$

Remark 6. For the Lebesgue scale measures $\Delta \mu_{1}(\theta)=\Delta \theta, \Delta$ $\mu_{2}(\eta)=\Delta \eta$, and $\xi=\xi^{*}$, Corollary 5 coincides with Corollary 3.3 in [10].

Now, considering Theorem 3 with $\Phi(x)=e^{x}$ and $f=\ln$ $\left(g^{\xi}\right)$, we get the following inviting result.

Corollary 7. Suppose the supposition of Theorem 3 is gratified only with $\Phi(x)=e^{x}$. If $0<\xi \leq \xi^{*}<\infty$, then inequality (32) yields the following result: 


$$
\begin{aligned}
& \left(\int_{\Omega_{1}} u(\theta)\left[e^{\xi / K(\theta) \int_{\Omega_{2}} k(\theta, \eta) \ln (g(\eta)) \Delta \mu_{2}(\eta)}\right]^{\xi^{*} / \xi} \Delta \mu_{1}(\theta)\right)^{1 / \xi^{*}} \\
& \quad \leq\left(\int_{\Omega_{2}} v(\eta)[g(\eta)]^{\xi} \Delta \mu_{2}(\eta)\right)^{1 / \xi} .
\end{aligned}
$$

Remark 8. For the Lebesgue scale measures $\Delta \mu_{1}(\theta)=\Delta \theta, \Delta$ $\mu_{2}(\eta)=\Delta \eta$, and $\xi=\xi^{*}$, Corollary 7 coincides with Corollary 3.4 in [10]. Also, in the special case $\xi=1$, Remark 8 coincides with Corollary 3.5 in [10].

Now, by using a special substitution, we obtain our central result; that is, if we replace $k(\theta, \eta)$ by $k(\theta, \eta) f_{2}(\eta)$ and $f$ by $f_{1} / f_{2}$, where $f_{j}: \Omega_{2} \rightarrow \mathbb{R}(j=1,2)$ are measurable functions, we obtain these results.

Theorem 9. Assume (H1) and (H2) and $v$ be defined on $\Omega_{2}$ by $v(\eta):=f_{2}(\eta)\left(\int_{\Omega_{1}} u(\theta)\left(\frac{k(\theta, \eta)}{g_{2}(\theta)}\right)^{\xi^{*} / \xi} \Delta \mu_{1}(\theta)\right)^{\xi / \xi^{*}}<\infty, \quad g_{2}(\theta) \neq 0$,

where $0<\xi \leq \xi^{*}<\infty$. If $\Phi \in C(I, \mathbb{R})$ is a positive convex, then the following inequality

$$
\begin{gathered}
\left(\int_{\Omega_{1}} u(\theta)\left[\Phi\left(\frac{g_{1}(\theta)}{g_{2}(\theta)}\right)\right]^{\xi^{*} / \xi} \Delta \mu_{1}(\theta)\right)^{1 / \xi^{*}} \\
\leq\left(\int_{\Omega_{2}} v(\eta) \Phi\left(\frac{f_{1}(\eta)}{f_{2}(\eta)}\right) \Delta \mu_{2}(\eta)\right)^{1 / \xi}
\end{gathered}
$$

is available for all measurable functions $f_{j}: \Omega_{2} \rightarrow \mathbb{R}(j=1,2)$ and

$$
g_{j}(\theta):=\int_{\Omega_{2}} k(\theta, \eta) f_{j}(\eta) \Delta \mu_{2}(\eta) \quad(j=1,2)
$$

Remark 10. Choosing $\mathbb{T}=\mathbb{R}$ in Theorem 9 , then inequality (40) reduces to inequality (12) established by Iqbal et al. (Theorem 2.1, [7]).

As a special case of Theorem 9 for $\xi=\xi^{*}$, we get the next corollary. Also, we note that the function $\Phi$ need not to be positive.

Corollary 11. Assume (H1) and (H2) and $v$ be defined on $\Omega_{2}$ by

$$
v(\eta):=f_{2}(\eta) \int_{\Omega_{1}} u(\theta) \frac{k(\theta, \eta)}{g_{2}(\theta)} \Delta \mu_{1}(\theta)<\infty, \quad g_{2}(\theta) \neq 0 .
$$

If $\Phi \in C(I, \mathbb{R})$ is a positive convex, then the following inequality

$$
\int_{\Omega_{1}} u(\theta) \Phi\left(\frac{g_{1}(\theta)}{g_{2}(\theta)}\right) \Delta \mu_{1}(\theta) \leq \int_{\Omega_{2}} v(\eta) \Phi\left(\frac{f_{1}(\eta)}{f_{2}(\eta)}\right) \Delta \mu_{2}(\eta)
$$

is available for all measurable functions $f_{j}: \Omega_{2} \rightarrow \mathbb{R}(j=1,2)$ and $g_{j}$ is defined by (41).

Corollary 12. Suppose the supposition of Theorem 9 is gratified only with $\Phi(x)=x^{\xi}$ for $\xi>1$. Then, inequality (40) yields the following result:

$$
\left(\int_{\Omega_{1}} u(\theta)\left(\frac{g_{1}(\theta)}{g_{2}(\theta)}\right)^{\xi^{*}} \Delta \mu_{1}(\theta)\right)^{1 / \xi^{*}} \leq\left(\int_{\Omega_{2}} v(\eta)\left(\frac{f_{1}(\eta)}{f_{2}(\eta)}\right)^{\xi} \Delta \mu_{2}(\eta)\right)^{1 / \xi}
$$

Corollary 13. Suppose the supposition of Theorem 9 is gratified only with $\Phi(x)=e^{x}$. If $0<\xi \leq \xi^{*}<\infty$, then inequality (40) yields the following result:

$$
\left(\int_{\Omega_{1}} u(\theta) e^{\xi^{*} g_{1}(\theta) / \xi g_{2}(\theta)} \Delta \mu_{1}(\theta)\right)^{1 / \xi^{*}} \leq\left(\int_{\Omega_{2}} v(\eta) e^{f_{1}(\eta) / f_{2}(\eta)} \Delta \mu_{2}(\eta)\right)^{1 / \xi} .
$$

Remark 14. Choosing $\mathbb{T}=\mathbb{R}$ in Corollary 11 , then (43) takes the form

$$
\int_{\Omega_{1}} u(\theta) \Phi\left(\frac{g_{1}(\theta)}{g_{2}(\theta)}\right) d \mu_{1}(\theta) \leq \int_{\Omega_{2}} v(\eta) \Phi\left(\frac{f_{1}(\eta)}{f_{2}(\eta)}\right) d \mu_{2}(\eta)
$$

where

$$
v(\eta):=f_{2}(\eta) \int_{\Omega_{1}} u(\theta) \frac{k(\theta, \eta)}{g_{2}(\theta)} d \mu_{1}(\theta)<\infty, \quad g_{2}(\theta) \neq 0,
$$

which is the same result due to Iqbal et al. (Corollary 2.2, [7]).

Corollary 15. For the Lebesgue diamond- $\alpha$ scale measures $\Delta$ $\mu_{1}(\theta)=\diamond_{\alpha} \theta, \Delta \mu_{2}(\eta)=\diamond_{\alpha} \eta, \Omega_{1}=\left[a_{1}, b_{1}\right]_{\mathbb{T}}$, and $\Omega_{2}=\left[c_{1}, d_{1}\right]_{\mathbb{T}}$, inequality (40) takes the form

$$
\left(\int_{a_{1}}^{b_{1}} u(\theta)\left[\Phi\left(\frac{g_{1}(\theta)}{g_{2}(\theta)}\right)\right]^{\xi^{*} / \xi} \diamond_{\alpha} \theta\right)_{1 / \xi^{*}} \leq\left(\int_{c_{1}}^{d_{1}} v(\eta) \Phi\left(\frac{f_{1}(\eta)}{f_{2}(\eta)}\right) \nabla_{\alpha} \eta\right)^{1 / \xi},
$$


where $0<\xi \leq \xi^{*}<\infty, \Phi \in C(I, \mathbb{R})$ is a positive convex, and

$$
\begin{aligned}
& v(\eta)=f_{2}(\eta)\left(\int_{a_{1}}^{b_{1}} u(\theta)\left(\frac{k(\theta, \eta)}{g_{2}(\theta)}\right)^{\xi^{*} / \xi} \diamond_{\alpha} \theta\right)^{\xi / \xi^{*}}<\infty, \quad g_{2}(\theta) \neq 0, \\
& g_{j}(\theta):=\int_{c_{1}}^{d_{1}} k(\theta, \eta) f_{j}(\eta) \diamond_{\alpha} \eta \quad(j=1,2) .
\end{aligned}
$$

Remark 16 . If we set $\xi=\xi^{*}=1$, then inequality (48) takes the form

$$
\int_{a_{1}}^{b_{1}} u(\theta) \Phi\left(\frac{g_{1}(\theta)}{g_{2}(\theta)}\right) \diamond_{\alpha} \theta \leq \int_{c_{1}}^{d_{1}} v(\eta) \Phi\left(\frac{f_{1}(\eta)}{f_{2}(\eta)}\right) \diamond_{\alpha} \eta
$$

which coincided with inequality (7) in [21].

Remark 17. If we set $\Phi(\theta)=-\ln |\theta|$, then inequality (50) takes the form

$$
\int_{a_{1}}^{b_{1}} u(\theta) \ln \left|\frac{g_{1}(\theta)}{g_{2}(\theta)}\right| \diamond_{\alpha} \theta \geq \int_{c_{1}}^{d_{1}} v(\eta) \ln \left|\frac{f_{1}(\eta)}{f_{2}(\eta)}\right| \diamond_{\alpha} \eta
$$

which coincided with Corollary 4 in [21].

Remark 18. In Remark 16, if we set $f_{2}(\eta)=1, g_{1}(\theta)=g(\theta)$, $f_{1}(\eta)=f(\eta)$, and $\Phi \in \mathrm{C}([0, \infty), \mathbb{R})$ be a convex function, then

$$
g_{2}(\theta):=\int_{c_{1}}^{d_{1}} k(\theta, \eta) \diamond_{\alpha} \eta=K(\theta), \quad \forall \theta \in\left[a_{1}, b_{1}\right]_{\mathbb{T}} .
$$

We assume that $K(\theta)>0$ and define

$$
v(\eta):=\int_{a_{1}}^{b_{1}} \frac{u(\theta) k(\theta, \eta)}{K(\theta)} \diamond_{\alpha} \theta, \quad \forall \theta \in\left[c_{1}, d_{1}\right]_{\mathbb{T}} .
$$

Then, inequality (50) takes the form

$$
\int_{a_{1}}^{b_{1}} u(\theta) \Phi\left(\frac{g(\theta)}{K(\theta)}\right) \diamond_{\alpha} \theta \leq \int_{c_{1}}^{d_{1}} v(\eta) \Phi(f(\eta)) \diamond_{\alpha} \eta
$$

which coincided with inequality (2.7) in [22] (Theorem 2.2).

Remark 19. Apply Remark 8 for $\Phi(\theta)=-\ln |\theta|$, which is convex with domain $(0, \infty)$. Then, inequality $(54)$ takes the form

$$
\int_{a_{1}}^{b_{1}} u(\theta) \ln \left(\frac{|g(\theta)|}{K(\theta)}\right) \diamond_{\alpha} \theta \geq \int_{c_{1}}^{d_{1}} v(\eta) \ln (|f(\eta)|) \diamond_{\alpha} \eta
$$

which coincided with inequality (2.10) in [22] (Corollary 2.3).
Remark 20. Apply Remark 18 for $\Phi(\theta)=e^{\theta}, \theta \geq 0$. Then, inequality (54) takes the form

$$
\int_{a_{1}}^{b_{1}} u(\theta) e^{g(\theta) / K(\theta)} \diamond_{\alpha} \theta \leq \int_{c_{1}}^{d_{1}} v(\eta) e^{f(\eta)} \diamond_{\alpha} \eta,
$$

which coincided with inequality (2.11) in [22] (Corollary 2.4).

Remark 21. If we set $\alpha=1$, then the delta version form of inequality (50) takes the form

$$
\int_{a_{1}}^{b_{1}} u(\theta) \Phi\left(\frac{g_{1}(\theta)}{g_{2}(\theta)}\right) \Delta \theta \leq \int_{c_{1}}^{d_{1}} v(\eta) \Phi\left(\frac{f_{1}(\eta)}{f_{2}(\eta)}\right) \Delta \eta,
$$

which coincided with inequality (8) in [21].

Remark 22. If we set $\alpha=0$, then the nabla version form of inequality (50) takes the form

$$
\int_{a_{1}}^{b_{1}} u(\theta) \Phi\left(\frac{g_{1}(\theta)}{g_{2}(\theta)}\right) \nabla \theta \leq \int_{c_{1}}^{d_{1}} v(\eta) \Phi\left(\frac{f_{1}(\eta)}{f_{2}(\eta)}\right) \nabla \eta,
$$

which coincided with inequality (9) in [21].

Remark 23. In particular, if $\mathbb{T}=\mathbb{R}$ and $\left[a_{1}, b_{1}\right]_{\mathbb{T}}=\left[c_{1}, d_{1}\right]_{\mathbb{T}}$, then inequality (50) takes the form

$$
\int_{a_{1}}^{b_{1}} u(\theta) \Phi\left(\frac{g_{1}(\theta)}{g_{2}(\theta)}\right) d \theta \leq \int_{c_{1}}^{d_{1}} v(\eta) \Phi\left(\frac{f_{1}(\eta)}{f_{2}(\eta)}\right) d \eta,
$$

where

$v(\eta):=f_{2}(\eta) \int_{a_{1}}^{b_{1}} u(\theta)\left(\frac{k(\theta, \eta)}{g_{2}(\theta)}\right) d \theta, \quad \forall \eta \in\left[a_{1}, b_{1}\right]_{\mathbb{T}}, g_{2}(\theta) \neq 0$,

$g_{j}(\theta):=\int_{a_{1}}^{b_{1}} k(\theta, \eta) f_{j}(\eta) d \eta, \quad \forall \theta \in\left[a_{1}, b_{1}\right]_{\mathbb{T}}, j=1,2$.

This result is given in $[23,24]$.

\section{Inequalities with Special Kernels}

The next theorem states the general result for Hardy's inequality in quotient.

Theorem 24. Suppose $0 \leq \xi \leq \xi^{*}<\infty$ and $u$ be a weight function. Define $v$ on $[e, l)_{\mathbb{T}}$ by

$$
v(\eta):=f_{2}(\eta)\left(\int_{\eta}^{l}\left(\int_{e}^{\sigma(\theta)} f_{2}(\eta) \Delta \eta\right)^{-\xi^{*} / \xi} u(\theta) \Delta \theta\right)^{\xi / \xi^{*}}<\infty .
$$

If $\Phi \in C(I, \mathbb{R})$ is a positive convex, then the following inequality 


$$
\begin{aligned}
& \left(\int_{e}^{l} u(\theta)\left(\Phi\left(\frac{\int_{e}^{\sigma(\theta)} f_{1}(\eta) \Delta \eta}{\int_{e}^{\sigma(\theta)} f_{2}(\eta) \Delta \eta}\right)\right)^{\xi^{*} / \xi} \Delta \theta\right)^{1 / \xi^{*}} \\
& \leq\left(\int_{e}^{l} v(\eta) \Phi\left(\frac{f_{1}(\eta)}{f_{2}(\eta)}\right) \Delta \eta\right)^{1 / \xi}
\end{aligned}
$$

is available for all measurable functions $f_{j}:[e, l)_{\mathbb{T}} \rightarrow \mathbb{R}$ $(j=1,2)$.

Proof. Rewrite inequality (40) with $\Omega_{1}=\Omega_{2}=[e, l)_{\mathbb{T}}, \Delta \mu_{1}(\theta)$ $=\Delta \theta$, and $\Delta \mu_{2}(\eta)=\Delta \eta$. Define the kernel $k:[e, l)_{\mathbb{T}} \times[e, l)_{\mathbb{T}}$ $\rightarrow \mathbb{R}$ by

$$
k(\theta, \eta):= \begin{cases}1, & \text { if } 0 \leq e \leq \eta \leq \sigma(\theta) \leq l \\ 0, & \text { if } \eta>\sigma(\theta)\end{cases}
$$

Then, $g_{j}$ in (41) takes the form

$$
g_{j}(\theta):=\int_{e}^{\sigma(\theta)} f_{j}(\eta) \Delta \eta
$$

Substituting $g_{j}(\theta)(j=1,2)$ in $(40)$, we acquired (47).

Corollary 25. If we apply Theorem 24 for $\Phi(\theta)=\theta^{\xi}, \xi \geq 1$, then (62) takes the form

$$
\left(\int_{e}^{l} u(\theta)\left(\frac{\int_{e}^{\sigma(\theta)} f_{1}(\eta) \Delta \eta}{\int_{e}^{\sigma(\theta)} f_{2}(\eta) \Delta \eta}\right)^{\xi^{*}} \Delta \theta\right)^{1 / \xi^{*}} \leq\left(\int_{e}^{l} v(\eta)\left(\frac{f_{1}(\eta)}{f_{2}(\eta)}\right)^{\xi} \Delta \eta\right)^{1 / \xi}
$$

where

$$
v(\eta):=f_{2}(\eta)\left(\int_{\eta}^{l}\left(\int_{e}^{\sigma(\theta)} f_{2}(\eta) \Delta \eta\right)^{-\xi^{*} / \xi} u(\theta) \Delta \theta\right)^{\xi / \xi^{*}}<\infty .
$$

Remark 26. If we put in Corollary $25 f_{2}(\eta)=1, \xi=\xi^{*}$, and $l$ $=\infty$, then we acquired Hardy's inequality on time scale

$$
\int_{e}^{\infty} \frac{u(\theta)}{\sigma(\theta)-e}\left(\int_{e}^{\sigma(\theta)} f_{1}(\eta) \Delta \eta\right)^{\xi} \Delta \theta \leq \int_{e}^{\infty} v(\eta) f_{1}^{\xi}(\eta) \Delta \eta
$$

where

$$
v(\eta):=\int_{\eta}^{\infty} \frac{u(\theta)}{\sigma(\theta)-e} \Delta \theta
$$

Remark 27. If we put in Theorem $24 \mathbb{T}=\mathbb{R}, e=0$, and $l=\infty$, we see that $\sigma(\theta)=\theta$ and then (62) takes the form

$$
\begin{aligned}
& \left(\int_{0}^{\infty} u(\theta)\left(\Phi\left(\frac{\int_{0}^{\theta} f_{1}(\eta) d \eta}{\int_{0}^{\theta} f_{2}(\eta) d \eta}\right)\right)^{\xi^{*} / \xi} d \theta\right)^{1 / \xi^{*}} \\
& \quad \leq\left(\int_{0}^{\infty} v(\eta) \Phi\left(\frac{f_{1}(\eta)}{f_{2}(\eta)}\right) d \eta\right)^{1 / \xi}
\end{aligned}
$$

where

$$
v(\eta):=f_{2}(\eta)\left(\int_{\eta}^{\infty}\left(\int_{0}^{\theta} f_{2}(\eta) d \eta\right)^{-\xi^{*} / \xi} u(\theta) d \theta\right)^{\xi / \xi^{*}}<\infty
$$

which is the same result due to Iqbal et al. (Theorem 2.4, [7]).

Example 1. If we put $\Phi(\theta)=\theta^{\xi}, \xi \geq 1$, and a particular weight function

$$
u(\theta):=\frac{1}{\theta^{2}}\left(\int_{e}^{\sigma(\theta)} f_{2}(\eta) \Delta \eta\right)^{\xi^{*} / \xi}, \quad \theta[\epsilon e, l)_{\mathbb{T}},
$$

in (62), we obtain

$$
v(\eta):=f_{2}(\eta)\left(\int_{\eta}^{l} \frac{1}{\theta^{2}} \Delta \theta\right)^{\xi / \xi^{*}}
$$

and inequality (62) becomes

$$
\begin{aligned}
& \left(\int_{e}^{l}\left(\int_{e}^{\sigma(\theta)} f_{1}(\eta) \Delta \eta\right)^{\xi^{*}}\left(\int_{e}^{\sigma(\theta)} f_{2}(\eta) \Delta \eta\right)^{\xi^{*}(1 / \xi-1)} \frac{\Delta \theta}{\theta^{2}}\right)^{1 / \xi^{*}} \\
& \leq\left(\int_{e}^{l}\left(\int_{\eta}^{l} \frac{1}{\theta^{2}} \Delta \theta\right)^{\xi / \xi^{*}} f_{1}^{\xi}(\eta) f_{2}^{1-\xi}(\eta) \Delta \eta\right)^{1 / \xi}
\end{aligned}
$$

For $\xi^{*}=\xi$, inequality $(73)$ reduces to

$$
\begin{gathered}
\int_{e}^{l}\left(\int_{e}^{\sigma(\theta)} f_{1}(\eta) \Delta \eta\right)^{\xi}\left(\int_{e}^{\sigma(\theta)} f_{2}(\eta) \Delta \eta\right)^{1-\xi} \frac{\Delta \theta}{\theta^{2}} \\
\leq \int_{e}^{l}\left(\int_{\eta}^{l} \frac{1}{\theta^{2}} \Delta \theta\right) f_{1}^{\xi}(\eta) f_{2}^{1-\xi}(\eta) \Delta \eta .
\end{gathered}
$$

Remark 28. When $\mathbb{T}=\mathbb{R}, e=0, l=\infty$, and $f_{2}(\eta)=1$, we see that $\sigma(\theta)=\theta$, and then (74) takes the form

$$
\int_{0}^{\infty}\left(\frac{1}{\theta} \int_{0}^{\theta} f_{1}(\eta) d \eta\right)^{\xi} \frac{d \theta}{\theta} \leq \int_{0}^{\infty} f_{1}^{\xi}(\eta) \frac{d \eta}{\eta}
$$

Now, for the convex function $\Phi \in \mathrm{C}(I, \mathbb{R})$ defined by $\Phi(\theta)=\exp (\theta)$, we can give the general form of PólyaKnopp's inequality in quotient. 
Theorem 29. Suppose $0 \leq \xi \leq \xi^{*}<\infty$ and $u$ be a weight function defined on $[e, l)_{\mathbb{T}}$. Define $v$ on $[e, l)_{\mathbb{T}}$ by

$v(\eta):=\ln f_{2}(\eta)\left(\int_{\eta}^{l}\left(\int_{e}^{\sigma(\theta)} \ln f_{2}(\eta) \Delta \eta\right)^{-\xi^{*} / \xi} u(\theta) \Delta \theta\right)^{\xi / \xi^{*}}<\infty$.

Then, the following inequality:

$$
\begin{aligned}
& \left(\int_{e}^{l} u(\theta)\left(\exp \left(\frac{\int_{e}^{\sigma(\theta)} \ln f_{1}(\eta) \Delta \eta}{\int_{e}^{\sigma(\theta)} \ln f_{2}(\eta) \Delta \eta}\right)\right)^{\xi / \xi^{*}} \Delta \theta\right)^{1 / \xi^{*}} \\
& \leq\left(\int_{e}^{l} v(\eta) \exp \left(\frac{\ln f_{1}(\eta)}{\ln f_{2}(\eta)}\right) \Delta \eta\right)^{1 / \xi}
\end{aligned}
$$

is available for all positive measurable functions $f_{j}:[e, l)_{\mathbb{T}}$ $\rightarrow \mathbb{R}(j=1,2)$.

Proof. We rewrite inequality (40) with $\Omega_{1}=\Omega_{2}=[e, l)_{\mathbb{T}}, \Delta$ $\mu_{1}(\theta)=\Delta \theta, \Delta \mu_{2}(\eta)=\Delta \eta$, and $\Phi: \mathbb{R}_{+} \rightarrow \mathbb{R}$ defined by $\Phi(\theta)$ $=\exp (\theta)$; we obtain

$\left(\int_{e}^{l} u(\theta)\left(\exp \left(\frac{g_{1}(\theta)}{g_{2}(\theta)}\right)\right)^{\xi^{*} / \xi} \Delta \theta\right)^{1 / \xi^{*}} \leq\left(\int_{e}^{l} v(\eta) \exp \left(\frac{f_{1}(\eta)}{f_{2}(\eta)}\right) \Delta \eta\right)^{1 / \xi}$.

Define $k(\theta, \eta)$ such as Theorem 24. Substituting $g_{j}(\theta)$ $(j=1,2)$ defined by $(64)$ in $(78)$, we acquired

$$
\begin{aligned}
& \left(\int_{e}^{l} u(\theta)\left(\exp \left(\frac{\int_{e}^{\sigma(\theta)} f_{1}(\eta) \Delta \eta}{\int_{e}^{\sigma(\theta)} f_{2}(\eta) \mathrm{i} \eta}\right)\right)^{\xi^{*} / \xi} \Delta \theta\right)^{1 / \xi^{*}} \\
& \leq\left(\int_{e}^{l} v(\eta) \exp \left(\frac{f_{1}(\eta)}{f_{2}(\eta)}\right) \Delta \eta\right)^{1 / \xi} .
\end{aligned}
$$

Replacing $f_{j}$ by $\ln f_{j}(j=1,2)$ in (79), we acquired (77).

Remark 30. If we put in Theorem $29 \mathbb{T}=\mathbb{R}, e=0$, and $l=\infty$, we see that $\sigma(\theta)=\theta$ and then (77) takes the form

$$
\begin{aligned}
& \left(\int_{0}^{\infty} u(\theta)\left(\exp \left(\frac{\int_{0}^{\theta} \ln f_{1}(\eta) d \eta}{\int_{0}^{\theta} \ln f_{2}(\eta) d \eta}\right)\right)^{\xi^{*} / \xi} d \theta\right)^{1 / \xi^{*}} \\
& \quad \leq\left(\int_{0}^{\infty} v(\eta) \exp \left(\frac{\ln f_{1}(\eta)}{\ln f_{2}(\eta)}\right) d \eta\right)^{1 / \xi}
\end{aligned}
$$

where

$$
v(\eta):=\ln f_{2}(\eta)\left(\int_{\eta}^{\infty}\left(\int_{0}^{\theta} \ln f_{2}(\eta) d \eta\right)^{-\xi^{*} / \xi} u(\theta) d \theta\right)^{\xi / \xi^{*}}<\infty
$$

which is the same result due to Iqbal et al. (Corollary 2.6, [7]).

Remark 31. If we put $f_{2}(\eta)=\exp (),. \xi=\xi^{*}$, and $l=\infty$ in Theorem 29, we have

$$
\int_{e}^{\infty} u(\theta) \exp \left(\frac{\int_{e}^{\sigma(\theta)} \ln f_{1}(\eta) \Delta \eta}{\sigma(\theta)-e}\right) \Delta \theta \leq \int_{e}^{\infty} v(\eta) f_{1}(\eta) \Delta \eta
$$

where

$$
v(\eta):=\int_{\eta}^{\infty} \frac{u(\theta)}{\sigma(\theta)-e} \Delta \theta<\infty
$$

Remark 32. In a private case of Remark 31 , when $\mathbb{T}=\mathbb{R}$ and $e=0$, we see that $\sigma(\theta)=\theta$, and inequality (82) reduces to

$$
\int_{0}^{\infty} u(\theta) \exp \left(\frac{\int_{0}^{\theta} \ln f_{1}(\eta) d \eta}{\theta}\right) d \theta \leq \int_{0}^{\infty} v(\eta) f_{1}(\eta) d \eta,
$$

where

$$
v(\eta):=\int_{\eta}^{\infty} \frac{u(\theta)}{\theta} d \theta<\infty
$$

Example 2. Particularly, for the weight function

$$
u(\theta):=\frac{1}{\theta^{2}}\left(\int_{e}^{\sigma(\theta)} \ln f_{2}(\eta) \Delta \eta\right)^{\xi^{*} / \xi}, \quad \theta \in[e, l)_{\mathbb{T}},
$$

in Theorem 29, we obtain

$$
v(\eta):=\left(\int_{\eta}^{l} \frac{1}{\theta^{2}} \Delta \theta\right)^{\xi / \xi^{*}} \ln f_{2}(\eta)
$$

and inequality (77) becomes

$$
\begin{aligned}
& \left(\int_{e}^{l} \frac{1}{\theta^{2}}\left(\int_{e}^{\sigma(\theta)} \ln f_{2}(\eta) \Delta \eta\right)^{\xi^{*} / \xi}\left(\exp \left(\frac{\int_{e}^{\sigma(\theta)} \ln f_{1}(\eta) \Delta \eta}{\int_{e}^{\sigma(\theta)} \ln f_{2}(\eta) \Delta \eta}\right)\right)^{\xi^{*} / \xi} \Delta \theta\right)^{1 / \xi^{*}} \\
& \leq\left(\int_{e}^{l}\left(\int_{\eta}^{l} \frac{1}{\theta^{2}} \Delta \theta\right)^{\xi / \xi^{*}} \ln f_{2}(\eta) \exp \left(\frac{\ln f_{1}(\eta)}{\ln f_{2}(\eta)}\right) \Delta \eta\right)^{1 / \xi} .
\end{aligned}
$$

Remark 33. If we put $\mathbb{T}=\mathbb{R}, \xi=\xi^{*}, f_{2}(\eta)=\exp (),. e=0$, and $l=\infty$ in (88), we see that $\sigma(\theta)=\theta$, and inequality (88) reduces to 


$$
\int_{0}^{\infty} \exp \left(\frac{1}{\theta} \int_{0}^{\theta} \ln f_{1}(\eta) d \eta\right) \frac{d \theta}{\theta} \leq \int_{0}^{\infty} f_{1}(\eta) \frac{d \eta}{\eta}
$$

The next general result is for Hardy-Hilbert's inequality.

Theorem 34. Suppose $0 \leq \xi \leq \xi^{*}<\infty$ and $u$ be a weight function defined on $[0, \infty)_{\mathbb{T}}$. Define $v$ on $[0, \infty)_{\mathbb{T}}$ by

$v(\eta):=f_{2}(\eta)\left(\int_{0}^{\infty} \frac{u(\theta)}{(\theta+\eta)^{s \xi^{*} / \xi}}\left(\int_{0}^{\infty} \frac{f_{2}(\eta)}{(\theta+\eta)^{s}} \Delta \eta\right)^{-\xi^{*} / \xi} \Delta \theta\right)^{\xi / \xi^{*}}<\infty$.

If $\Phi \in C(I, \mathbb{R})$ is a positive convex, then the following inequality

$$
\begin{aligned}
& \left(\int_{0}^{\infty} u(\theta)\left(\Phi\left(\frac{\int_{0}^{\infty}\left(f_{1}(\eta) /(\theta+\eta)^{s}\right) \Delta \eta}{\int_{0}^{\infty}\left(f_{2}(\eta) /(\theta+\eta)^{s}\right) \Delta \eta}\right)\right)^{\xi^{*} / \xi} \Delta \theta\right)^{1 / \xi^{*}} \\
& \quad \leq\left(\int_{0}^{\infty} v(\eta) \Phi\left(\frac{f_{1}(\eta)}{f_{2}(\eta)}\right) \Delta \eta\right)^{1 / \xi}
\end{aligned}
$$

is available for all measurable functions $f_{j}:[0, \infty)_{\mathbb{\pi}} \rightarrow \mathbb{R}$ $(j=1,2)$.

Proof. We rewrite (40) with $\Omega_{1}=\Omega_{2}=[0, \infty)_{\mathbb{T}}, \Delta \mu_{1}(\theta)=\Delta \theta$, and $\Delta \mu_{2}(\eta)=\Delta \eta$. Define the kernel $k:[0, \infty)_{\mathbb{T}} \times[0, \infty)_{\mathbb{T}} \rightarrow \mathbb{R}$ by

$$
k(\theta, \eta):=\frac{(\eta / \theta)^{s-2 / \xi}}{(\theta+\eta)^{s}}, \quad \xi>1 .
$$

Then, $g_{j}$ defined in (64) becomes

$g_{j}(\theta):=\int_{0}^{\infty}\left(\frac{\eta}{\theta}\right)^{s-2 / \xi}(\theta+\eta)^{-s} f_{j}(\eta) \Delta \eta:=\theta^{2-s / \xi} \int_{0}^{\infty} \eta^{s-2 / \xi} \frac{f_{j}(\eta)}{(\theta+\eta)^{s}} \Delta \eta$.

Substituting $g_{j}(\theta)(j=1,2)$ in (40), we acquired

$$
\begin{aligned}
& \left(\int_{0}^{\infty} u(\theta)\left(\Phi\left(\frac{\int_{0}^{\infty} \eta^{s-2 / \xi}\left(f_{1}(\eta) /(\theta+\eta)^{s}\right) \Delta \eta}{\int_{0}^{\infty} \eta^{s-2 / \xi}\left(f_{2}(\eta) /(\theta+\eta)^{s}\right) \Delta \eta}\right)\right)^{\xi^{*} / \xi} \Delta \theta\right)^{1 / \xi^{*}} \\
& \quad \leq\left(\int_{0}^{\infty} v(\eta) \Phi\left(\frac{f_{1}(\eta)}{f_{2}(\eta)}\right) \Delta \eta\right)^{1 / \xi} .
\end{aligned}
$$
(91).
Remark 35. If we put in Theorem $34 \mathbb{T}=\mathbb{R}$, then (91) takes the form

$$
\begin{aligned}
& \left(\int_{0}^{\infty} u(\theta)\left(\Phi\left(\frac{\int_{0}^{\infty}\left(f_{1}(\eta) /(\theta+\eta)^{s}\right) d \eta}{\int_{0}^{\infty}\left(f_{2}(\eta) /(\theta+\eta)^{s}\right) d \eta}\right)\right)^{\xi^{*} / \xi} d \theta\right)^{1 / \xi^{*}} \\
& \quad \leq\left(\int_{0}^{\infty} v(\eta) \Phi\left(\frac{f_{1}(\eta)}{f_{2}(\eta)}\right) d \eta\right)^{1 / \xi}
\end{aligned}
$$

where

$v(\eta):=f_{2}(\eta)\left(\int_{0}^{\infty} \frac{u(\theta)}{(\theta+\eta)^{s \xi^{*} / \xi}}\left(\int_{0}^{\infty} \frac{f_{2}(\eta)}{(\theta+\eta)^{s}} d \eta\right)^{-\xi^{*} / \xi} d \theta\right)^{\xi / \xi^{*}}<\infty$,

which is the same result due to Iqbal et al. (Theorem 2.8, [7]).

Example 3. For $0<\alpha<\left(s \xi^{*}\right) / \xi$ and taking the particular weight function

$$
u(\theta):=\theta^{\alpha-1}\left(\int_{0}^{\infty} \frac{f_{2}(\eta)}{(\theta+\eta)^{s}} \Delta \eta\right)^{\xi^{*} / \xi}, \quad \theta \in[0, \infty)_{\mathbb{T}},
$$

we obtain,

$$
v(\eta):=f_{2}(\eta)\left(\int_{0}^{\infty} \frac{\theta^{\alpha-1}}{(\theta+\eta)^{s \xi^{*} / \xi}} \Delta \theta\right)^{\xi / \xi^{*}} .
$$

Let the function $\Phi:[0, \infty) \rightarrow \mathbb{R}$ be defined by $\Phi(\theta)=\theta^{\xi}$; then, inequality (91) becomes

$$
\begin{aligned}
& \left(\int_{0}^{\infty} \theta^{\alpha-1}\left(\int_{0}^{\infty} \frac{f_{1}(\eta)}{(\theta+\eta)^{s}} \Delta \eta\right)^{\xi^{*}}\left(\int_{0}^{\infty} \frac{f_{2}(\eta)}{(\theta+\eta)^{s}} \Delta \eta\right)^{\xi^{*}(1 / \xi-1)} \Delta \theta\right)^{1 / \xi^{*}} \\
& \quad \leq\left(\int_{0}^{\infty}\left(\int_{0}^{\infty} \frac{\theta^{\alpha-1}}{(\theta+\eta)^{s \xi^{*} / \xi}} \Delta \theta\right)^{\xi^{*} / \xi}\left(f_{1}(\eta)\right)^{\xi}\left(f_{2}(\eta)\right)^{1-\xi} \Delta \eta\right)^{1 / \xi} .
\end{aligned}
$$

Remark 36. When $\mathbb{T}=\mathbb{R}$ in Example 3, we see that

$$
v(\eta):=\eta^{\alpha \xi / \xi^{*}-s} f_{2}(\eta)\left(B\left(\alpha, \frac{s \xi^{*}}{\xi}-\alpha\right)\right)^{\xi / \xi^{*}}
$$

and then (99) takes the form

$$
\begin{aligned}
& \left(\int_{0}^{\infty} \theta^{\alpha-1}\left(\int_{0}^{\infty} \frac{f_{1}(\eta)}{(\theta+\eta)^{s}} d \eta\right)^{\xi^{*}}\left(\int_{0}^{\infty} \frac{f_{2}(\eta)}{(\theta+\eta)^{s}} d \eta\right)^{\xi^{*}(1 / \xi-1)} d \theta\right)^{1 / \xi^{*}} \\
& \quad \leq\left(B\left(\alpha, \frac{s \xi^{*}}{\xi}-\alpha\right)\right)^{1 / \xi^{*}}\left(\int_{0}^{\infty} \eta^{\alpha \xi / \xi^{*}-s}\left(f_{1}(\eta)\right)^{\xi}\left(f_{2}(\eta)\right)^{1-\xi} d \eta\right)^{1 / \xi},
\end{aligned}
$$


where $B$ is the beta function, which is the same result due to Iqbal et al. (Example 2.9, [7]).

In the forthcoming theorem, we give the HardyLittlewood-Pólya inequality in quotient.

Theorem 37. Suppose $0 \leq \xi \leq \xi^{*}<\infty$ and $u$ be a weight function defined on $[0, \infty)_{\mathbb{T}}$. Define $v$ on $[0, \infty)_{\mathbb{T}}$ by

$v(\eta):=f_{2}(\eta)\left(\int_{0}^{\infty} \frac{u(\theta)}{(\max \{\theta, \eta\})^{s \xi^{*} / \xi}}\left(\int_{0}^{\infty} \frac{f_{2}(\eta)}{(\max \{\theta, \eta\})^{s}} \Delta \eta\right)^{-\xi^{*} / \xi} \Delta \theta\right)^{\xi / \xi^{*}}<\infty$.

If $\Phi \in C(I, \mathbb{R})$ is a positive convex, then the following inequality

$$
\begin{aligned}
& \left(\int_{0}^{\infty} u(\theta)\left(\Phi\left(\frac{\int_{0}^{\infty}\left(f_{1}(\eta) /(\max \{\theta, \eta\})^{s}\right) \Delta \eta}{\int_{0}^{\infty}\left(f_{2}(\eta) /(\max \{\theta, \eta\})^{s}\right) \Delta \eta}\right)\right)^{\xi^{*} / \xi} \Delta \theta\right)^{1 / \xi^{*}} \\
& \quad \leq\left(\int_{0}^{\infty} v(\eta) \Phi\left(\frac{f_{1}(\eta)}{f_{2}(\eta)}\right) \Delta \eta\right)^{1 / \xi},
\end{aligned}
$$

is available for all measurable functions $f_{j}:[0, \infty)_{\mathbb{T}} \rightarrow \mathbb{R}$ $(j=1,2)$.

Proof. Rewrite inequality (40) with $\Omega_{1}=\Omega_{2}=[0, \infty)_{\mathbb{T}}, \Delta \mu_{1}$ $(\theta)=\Delta \theta$, and $\Delta \mu_{2}(\eta)=\Delta \eta$. Define the kernel $\Phi:[0, \infty)_{\mathbb{T}} \times$ $[0, \infty)_{\mathbb{T}} \rightarrow \mathbb{R}$ by

$$
k(\theta, \eta):=\left(\frac{\eta}{\theta}\right)^{s-2 / \xi}(\max \{\theta, \eta\})^{-s}
$$

Then, $g_{j}$ defined in (64) becomes

$g_{j}(\theta):=\int_{0}^{\infty} \frac{(\eta / \theta)^{s-2 / \xi}}{(\max \{\theta, \eta\})^{s}} f_{j}(\eta) \Delta \eta:=\theta^{2-s / \xi} \int_{0}^{\infty} \eta^{s-2 / \xi} \frac{f_{j}(\eta)}{(\max \{\theta, \eta\})^{s}} \Delta \eta$.

Substituting $g_{j}(\theta)(j=1,2)$ in $(40)$, we acquired

$$
\begin{aligned}
& \left(\int_{0}^{\infty} u(\theta)\left(\Phi\left(\frac{\int_{0}^{\infty} \eta^{s-2 / \xi}\left(\left(f_{1}(\eta) / \max \{\theta, \eta\}\right)^{s}\right) \Delta \eta}{\int_{0}^{\infty} \eta^{s-2 / \xi}\left(f_{2}(\eta) /(\max \{\theta, \eta\})^{s}\right) \Delta \eta}\right)\right)^{\xi^{*} / \xi} \Delta \theta\right)^{1 / \xi^{*}} \\
& \quad \leq\left(\int_{0}^{\infty} v(\eta) \Phi\left(\frac{f_{1}(\eta)}{f_{2}(\eta)}\right) \Delta \eta\right)^{1 / \xi} .
\end{aligned}
$$
(103).
Remark 38. When $\mathbb{T}=\mathbb{R}$ in Theorem 37, then (103) takes the form

$$
\begin{aligned}
& \left(\int_{0}^{\infty} u(\theta)\left(\Phi\left(\frac{\int_{0}^{\infty}\left(\left(f_{1}(\eta) / \max \{\theta, \eta\}\right)^{s}\right) d \eta}{\int_{0}^{\infty}\left(f_{2}(\eta) /(\max \{\theta, \eta\})^{s}\right) d \eta}\right)\right)^{\xi^{*} / \xi} d \theta\right)^{1 / \xi^{*}} \\
& \quad \leq\left(\int_{0}^{\infty} v(\eta) \Phi\left(\frac{f_{1}(\eta)}{f_{2}(\eta)}\right) d \eta\right)^{1 / \xi},
\end{aligned}
$$

where

$v(\eta):=f_{2}(\eta)\left(\int_{0}^{\infty} \frac{u(\theta)}{(\max \{\theta, \eta\})^{s \xi^{*} / \xi}}\left(\int_{0}^{\infty} \frac{f_{2}(\eta)}{(\max \{\theta, \eta\})^{s}} d \eta\right)^{-\xi^{*} / \xi} d \theta\right)^{\xi / \xi^{*}}<\infty$,

which is the same result due to Iqbal et al. (Theorem 2.10, [7]).

Example 4. For $0<\alpha<\left(s \xi^{*}\right) / \xi$ and taking the particular weight function

$$
u(\theta):=\theta^{\alpha-1}\left(\int_{0}^{\infty} \frac{f_{2}(\eta)}{(\max \{\theta, \eta\})^{s}} \Delta \eta\right)^{\xi / \xi^{*}}, \quad \theta \in[0, \infty)_{\mathbb{T}},
$$

we obtain

$$
v(\eta):=f_{2}(\eta)\left(\int_{0}^{\infty} \frac{\theta^{\alpha-1}}{(\max \{\theta, \eta\})^{\xi \xi^{*} / \xi}} \Delta \theta\right)^{\xi^{*} / \xi} .
$$

Let the function $\Phi:[0, \infty) \rightarrow \mathbb{R}$ be defined by $\Phi(\theta)=\theta^{\xi}$, then inequality (103) becomes

$$
\begin{aligned}
& \left(\int_{0}^{\infty} \theta^{\alpha-1}\left(\int_{0}^{\infty} \frac{f_{1}(\eta)}{(\max \{\theta, \eta\})^{s}} \Delta \eta\right)^{\xi^{*}}\left(\int_{0}^{\infty} \frac{f_{2}(\eta)}{(\max \{\theta, \eta\})^{s}} \Delta \eta\right)^{\xi^{*}\left(1 / \xi^{-1}-1\right)} \Delta \theta\right)^{1 / \xi^{*}} \\
& \leq\left(\int_{0}^{\infty}\left(\int_{0}^{\infty} \frac{\theta^{\alpha-1}}{(\max \{\theta, \eta\})^{s \xi^{*} / \xi}} \Delta \theta\right)^{\xi \xi / \xi^{*}}\left(f_{1}(\eta)\right)^{\xi}\left(f_{2}(\eta)\right)^{1-\xi} \Delta \eta\right)^{1 / \xi} .
\end{aligned}
$$

Remark 39. When $\mathbb{T}=\mathbb{R}$ in Example 4, we see that

$$
v(\eta):=\eta^{\alpha \xi / \xi^{*}-s} f_{2}(\eta)\left(\frac{s \xi^{*}}{\alpha\left(s \xi^{*}-\alpha \xi\right)}\right)^{\xi / \xi^{*}}
$$


and then inequality (111) becomes

$$
\begin{aligned}
& \left(\int_{0}^{\infty} \theta^{\alpha-1}\left(\int_{0}^{\infty} \frac{f_{1}(\eta)}{(\max \{\theta, \eta\})^{s}} d \eta\right)^{\xi^{*}}\left(\int_{0}^{\infty} \frac{f_{2}(\eta)}{(\max \{\theta, \eta\})^{s}} d \eta\right)^{\xi^{*}(1 / \xi-1)} d \theta\right)^{1 / \xi^{*}} \\
& \quad \leq\left(\frac{s \xi^{*}}{\alpha\left(s \xi^{*}-\alpha \xi\right)}\right)^{1 / \xi^{*}}\left(\int_{0}^{\infty} \eta^{\alpha \xi / \xi^{*}-s} f_{1}^{\xi}(\eta) f_{2}^{1-\xi}(\eta) d \eta\right)^{1 / \xi}
\end{aligned}
$$

which is the same result due to Iqbal et al. (Example 2.11, [7]).

Now, we give the result for the Hardy-Hilbert-type inequality in quotient.

Theorem 40. Let $0 \leq \xi \leq \xi^{*}<\infty$ and $u$ be a weight function defined on $[0, \infty)_{\mathbb{T}}$. Define $v$ on $[0, \infty)_{\mathbb{T}}$ by

$v(\eta):=f_{2}(\eta)\left(\int_{0}^{\infty} u(\theta)\left(\frac{\ln \eta-\ln \theta}{\eta-\theta}\right)^{\xi^{*} / \xi}\left(\int_{0}^{\infty} \frac{\ln \eta-\ln \theta}{\eta-\theta} f_{2}(\eta) \Delta \eta\right)^{-\xi^{*} / \xi} \Delta \theta\right)^{\xi / \xi^{*}}<\infty . \quad v(\eta):=f_{2}(\eta)\left(\int_{0}^{\infty} u(\theta)\left(\frac{\ln \eta-\ln \theta}{\eta-\theta}\right)^{\xi^{*} / \xi}\left(\int_{0}^{\infty} \frac{\ln \eta-\ln \theta}{\eta-\theta} f_{2}(\eta) d \eta\right)^{-\xi^{*} / \xi} d \theta\right)^{\xi / \xi^{*}}<\infty$.

If $\Phi \in C(I, \mathbb{R})$ is a positive convex, then the following inequality

$$
\begin{aligned}
& \left(\int_{0}^{\infty} u(\theta)\left(\Phi\left(\frac{\int_{0}^{\infty}((\ln \eta-\ln \theta) / \eta-\theta) f_{1}(\eta) \Delta \eta}{\int_{0}^{\infty}((\ln \eta-\ln \theta) / \eta-\theta) f_{2}(\eta) \Delta \eta}\right)\right)^{\xi^{*} / \xi} \Delta \theta\right)^{1 / \xi^{*}} \\
& \quad \leq\left(\int_{0}^{\infty} v(\eta) \Phi\left(\frac{f_{1}(\eta)}{f_{2}(\eta)}\right) \Delta \eta\right)^{1 / \xi}
\end{aligned}
$$

is available for all measurable functions $f_{j}:[0, \infty)_{\mathbb{T}} \rightarrow \mathbb{R}$ $(j=1,2)$.

Proof. Rewrite inequality (40) with $\Omega_{1}=\Omega_{2}=[0, \infty)_{\mathbb{T}}, \Delta \mu_{1}$ $(\theta)=\Delta \theta$, and $\Delta \mu_{2}(\eta)=\Delta \eta$. For $\alpha \in(0,1)$, we define the kernel $k:[0, \infty)_{\mathbb{T}} \times[0, \infty)_{\mathbb{T}} \rightarrow \mathbb{R}$ by

$$
k(\theta, \eta):=\frac{\ln (\eta / \theta)}{\eta-\theta}\left(\frac{\theta}{\eta}\right)^{\alpha}
$$

Then, $g_{j}$ defined in (64) takes the form

$g_{j}(\theta):=\int_{0}^{\infty} \frac{\ln \eta-\ln \theta}{\eta-\theta}\left(\frac{\theta}{\eta}\right)^{\alpha} f_{j}(\eta) \Delta \eta:=\theta^{\alpha} \int_{0}^{\infty} \frac{\ln \eta-\ln \theta}{\eta-\theta} \eta^{-\alpha} f_{j}(\eta) \Delta \eta$.

Substituting $g_{j}(\theta)(j=1,2)$ in $(40)$, we acquired

$$
\begin{aligned}
& \left(\int_{0}^{\infty} u(\theta)\left(\Phi\left(\frac{\int_{0}^{\infty}((\ln \eta-\ln \theta) / \eta-\theta) \eta^{-\alpha} f_{1}(\eta) \Delta \eta}{\int_{0}^{\infty}((\ln \eta-\ln \theta) / \eta-\theta) \eta^{-\alpha} f_{2}(\eta) \mathrm{i} \eta}\right)\right)^{\xi^{*} / \xi} \Delta \theta\right)^{1 / \xi^{*}} \\
& \quad \leq\left(\int_{0}^{\infty} v(\eta) \Phi\left(\frac{f_{1}(\eta)}{f_{2}(\eta)}\right) \Delta \eta\right)^{1 / \xi} .
\end{aligned}
$$

Writing $f_{j}(\eta)$ instead of $\eta^{-\alpha} f_{j}(\eta)$ in (118), we obtain (115).

Remark 41 . If we put $\mathbb{T}=\mathbb{R}$ in Theorem 34 , we acquired the Hardy-Hilbert-type inequality in [7] (Theorem 2.12):

$$
\begin{aligned}
& \left(\int_{0}^{\infty} u(\theta)\left(\Phi\left(\frac{\int_{0}^{\infty}((\ln \eta-\ln \theta) / \eta-\theta) f_{1}(\eta) d \eta}{\int_{0}^{\infty}((\ln \eta-\ln \theta) / \eta-\theta) f_{2}(\eta) d \eta}\right)\right)^{\xi^{*} / \xi} d \theta\right)^{1 / \xi^{*}} \\
& \quad \leq\left(\int_{0}^{\infty} v(\eta) \Phi\left(\frac{f_{1}(\eta)}{f_{2}(\eta)}\right) d \eta\right)^{1 / \xi},
\end{aligned}
$$

where

Example 5. For $\alpha \in(0,1)$ and for

$$
u(\theta):=\theta^{-\alpha}\left(\frac{\ln (\eta / \theta)}{\eta-\theta} f_{2}(\eta) \Delta \eta\right)^{\xi^{*} / \xi}, \quad \theta \in[0, \infty)_{\mathbb{T}}
$$

in (114), we obtain

$$
v(\eta):=\eta^{(1-\alpha) \xi / \xi^{*}-1} f_{2}(\eta) C
$$

where

$$
C:=\left(\int_{0}^{\infty} z^{-\alpha}\left(\frac{\ln z}{z-1}\right)^{\xi^{*} / \xi} \Delta z\right)^{\xi / \xi^{*}} .
$$

Let $\xi \geq 1$ and the function $\Phi:[0, \infty) \rightarrow \mathbb{R}$ be defined by $\Phi(\theta)=\theta^{\xi}$. Then, inequality (115) becomes

$$
\begin{aligned}
& \left(\int_{0}^{\infty} \theta^{-\alpha}\left(\int_{0}^{\infty} \frac{\ln (\eta / \theta)}{\eta-\theta} f_{1}(\eta) \Delta \eta\right)^{\xi^{*}}\left(\int_{0}^{\infty} \frac{\ln (\eta / \theta)}{\eta-\theta} f_{2}(\eta) \Delta \eta\right)^{\xi^{*}(1 / \xi-1)} \Delta \theta\right)^{1 / \xi^{*}} \\
& \quad \leq\left(C \int_{0}^{\infty} \eta^{(1-\alpha) \xi / \xi^{*}-1} f_{1}^{\xi}(\eta) f_{2}^{1-\xi}(\eta) \Delta \eta\right)^{1 / \xi} .
\end{aligned}
$$

Remark 42. When $\mathbb{T}=\mathbb{R}$ in Example 5, we see that

$$
\begin{aligned}
& u(\theta):=\theta^{-\alpha}\left(\frac{\ln (\eta / \theta)}{\eta-\theta} f_{2}(\eta) d \eta\right)^{\xi^{*} / \xi}, \quad \theta \in[0, \infty) \\
& v(\eta):=\eta^{(1-\alpha) \xi / \xi^{*}-1} f_{2}(\eta) C
\end{aligned}
$$

where

$$
C:=\left(\int_{0}^{\infty} z^{-\alpha}\left(\frac{\ln z}{z-1}\right)^{\xi^{*} / \xi} d z\right)^{\xi / \xi^{*}}
$$


and then inequality (124) becomes

$$
\begin{aligned}
& \left(\int_{0}^{\infty} \theta^{-\alpha}\left(\int_{0}^{\infty} \frac{\ln (\eta / \theta)}{\eta-\theta} f_{1}(\eta) d \eta\right)^{\xi^{*}}\left(\int_{0}^{\infty} \frac{\ln (\eta / \theta)}{\eta-\theta} f_{2}(\eta) d \eta\right)^{\xi^{*}\left(\xi^{-1}-1\right)} d \theta\right)^{1 / \xi^{*}} \\
& \quad \leq\left(C \int_{0}^{\infty} \eta^{(1-\alpha) \xi / \xi^{*}-1} f_{1}^{\xi}(\eta) f_{2}^{1-\xi}(\eta) d \eta\right)^{1 / \xi}
\end{aligned}
$$

which is the same result due to Iqbal et al. (Example 2.13, [7]).

\section{Conclusions}

In this paper, with the help of special kernels, we obtained some nouveau forms for Hardy's and related inequalities on time scales in quotients and also we presented some recent results for the Pólya-Knopp-, Hardy-Hilbert-, Hardy-Littlewood-Pólya-type inequalities. Furthermore, we got some continuous inequalities as special cases of the obtained dynamic inequalities.

\section{Data Availability}

No data were used to support this study.

\section{Conflicts of Interest}

The authors declare that they have no conflicts of interest.

\section{Authors' Contributions}

All authors contributed equally. All the authors read and approved the final manuscript.

\section{Acknowledgments}

This research was funded by the Deanship of Scientific Research at Princess Nourah Bint Abdulrahman University through the Fast-track Research Funding Program.

\section{References}

[1] G. H. Hardy, "Notes on some points in the integral calculus. LX. An inequality between integrals," Messenger of Math, vol. 54, pp. 150-156, 1925.

[2] K. Knopp, “Über Reihen mit positiven Gliedern," Journal of the London Mathematical Society, vol. 3, pp. 205-311, 1928.

[3] S. Kaijser, L. E. Persson, and A. Öberg, "On Carleman and Knopp's inequalities," Journal of Approximation Theory, vol. 117, no. 1, pp. 140-151, 2002.

[4] A. Čižmešija, J. Pečarić, and L.-E. Persson, "On strengthened Hardy and Pólya-Knopp's inequalities," Journal of Approximation Theory, vol. 125, no. 1, pp. 74-84, 2003.

[5] S. Kaijser, L. Nikolova, L. E. Persson, and A. Wedestig, "Hardy type inequalities via convexity," Mathematical Inequalities \& Applications, vol. 8, no. 3, pp. 403-417, 2005.

[6] K. Krulić, J. Pečarić, and L.-E. Persson, "Some new Hardy type inequalities with general kernels," Mathematical Inequalities \& Applications, vol. 12, no. 3, pp. 473-485, 1998.
[7] S. Iqbal, K. Krulic-Himmelreich, and J. Pečaric, "Hardy's and related inequalities in quotients," Acta Mathematica Universitatis Comenianae, vol. 83, no. 2, pp. 195-207, 2014.

[8] P. Řehák, "Hardy inequality on time scales and its application to half-linear dynamic equations," Journal of Inequalities and Applications, vol. 2005, no. 5, Article ID 942973, 2005.

[9] U. M. Özkan and H. Yildirim, "Hardy-Knopp type inequalities on time scales," Dynamic Systems and Applications, vol. 17, pp. 477-486, 2008.

[10] M. Bohner, A. Nosheen, J. Pečaríc, and A. Younus, "Some dynamic Hardy-type inequalities with general kernel," Journal of Mathematical Inequalities, vol. 8, no. 1, pp. 185-199, 2007.

[11] S. Abramovich, K. Krulić, J. Pečarić, and L. E. Persson, "Some new refined Hardy type inequalities with general kernels and measures," Aequationes Mathematicae, vol. 79, no. 1-2, pp. 157-172, 2010.

[12] R. P. Agarwal, R. R. Mahmoud, S. H. Saker, and Ç. Tunc, "New generalizations of Németh-Mohapatra type inequalities on time scales," Acta Mathematica Hungarica, vol. 152, no. 2, pp. 383-403, 2017.

[13] A. M. Ahmed, G. AlNemer, M. Zakarya, and H. M. Rezk, "Some dynamic inequalities of Hilbert's type," Journal of Function Spaces, vol. 2020, Article ID 4976050, 13 pages, 2020.

[14] S. H. Saker, A. M. Ahmed, H. M. Rezk, D. O’Regan, and R. P. Agarwal, "New Hilbert's dynamic inequalities on time scales," Mathematical Inequalities \& Applications, vol. 20, no. 40, pp. 1017-1039, 2017.

[15] S. H. Saker, H. M. Rezk, D. O’Regan, and R. P. Agarwal, “A variety of inverse Hilbert type inequality on time scales," Dynamics of Continuous, Discrete and Impulsive Systems Series A: Mathematical Analysis, vol. 24, pp. 347-373, 2017.

[16] S. H. Saker, H. M. Rezk, and M. Krnić, "More accurate dynamic Hardy-type inequalities obtained via superquadraticity," Revista de la Real Academia de Ciencias Exactas, Físicas y Naturales. Serie A. Matemáticas, vol. 113, no. 3, pp. 2691-2713, 2019.

[17] S. H. Saker, H. M. Rezk, I. Abohela, and D. Baleanu, "Refinement multidimensional dynamic inequalities with general kernels and measures," Journal of Inequalities and Applications, vol. 2019, no. 1, 2019.

[18] S. H. Saker, Ç. Tunc, and R. R. Mahmoud, "New CarlsonBellman and Hardy-Littlewood dynamic inequalities," Mathematical Inequalities \& Applications, vol. 21, no. 4, pp. 967-983, 2018.

[19] M. Bohner and A. Peterson, Dynamic Equations on Time Scales: An Introduction with Applications, Birkhäuser Boston, 2001.

[20] M. Bohner and A. Peterson, Eds., Advances in Dynamic Equations on Time Scales, Birkhäuser Boston, 2003.

[21] M. J. S. Sahir, "Dynamic inequalities for convex functions harmonized on time scales," Joural of Applied Mathematics and Physics, vol. 5, no. 12, pp. 2360-2370, 2017.

[22] G. A. Anastassiou, "Integral operator inequalities on time scales," International Journal of Difference Equations, vol. 7, pp. 111-137, 2012.

[23] D. S. Mitrinović, "Generalizations of two inequalities of Godunova and Levin," Bulletin of the Polish Academy of Sciences, vol. 36, pp. 645-648, 1988.

[24] J. E. Peĉarić, F. Proschan, and Y. L. Tong, Convex Functions, Partial Orderings and Statistical Applications, vol. 187 of Mathematics in Science and Engineering, Academic Press, Boston, 1992. 